\title{
Enhancing Diabetes Self-Management Education and Psychological Services for Veterans With Comorbid Chronic Health and Mental Health Conditions
}

\author{
Naomi S. Kane, PhD; Lindsey E. Bloor, PhD, ABPP; and Jamie Michaels, MS, RD, CDE
}

Background: Veterans experience a higher prevalence of type 2 diabetes mellitus (T2DM) compared with the rate of their civilian counterparts. Veterans may experience vulnerability to chronic stress, in particular comorbid mental health conditions, and may not benefit from traditional diabetes education.

Methods: This study evaluated clinical and psychological measures among veterans engaged in health psychology services. Individualized motivational interviewing and cognitive behavioral interventions were provided to address T2DM distress and promote veterans' diabetes self-management. Pre-/postobjective and self-report measures were evaluated for clinical relevancy and statistically significant changes.

Results: The sample consisted of 13 older adults: mean age 62.8 years; 12 were male and 9 were prescribed insulin. More than half had comorbid hypertension, hyperlipidemia, and/or a diagnosis of chronic pain. Eleven participants were diagnosed with a mental health disorder. Baseline measures indicated mild depressive symptoms, mild anxiety symptoms, and moderate levels of T2DM distress. Postintervention reductions were shown for T2DM distress; emotional burden, and regimen-related distress, depressive symptoms, and enhanced diabetes empowerment.

Conclusions: Veterans with comorbid conditions may benefit from individualized psychology services that offer cognitive behavioral strategies for self-management of T2DM-related distress, integrated with traditional primary care and diabetes education.
Author affiliations can be found at the end of the article.

Correspondence: Naomi Kane (naomikanephd@gmail.com)

Fed Pract. 2021;38(4). Published online April 14, 2021. doi: $10.12788 / \mathrm{fp} .0106$
V eterans have a higher prevalence of type 2 diabetes mellitus (T2DM) when compared with their civilian counterparts with an overall prevalence rate of $25 \% .^{1}$ This higher prevalence is similar to other major chronic health conditions, including heart disease and arthritis, with additional costs for disease self-management. ${ }^{2}$ Psychological and behavioral change strategies are a principal means of limiting the severity and even restoring function once T2DM is diagnosed. ${ }^{3}$ More broadly, there is mounting evidence that addressing distress and behavior change are important across many conditions, particularly T2DM. ${ }^{4}$ Therefore, the US Department of Veterans Affairs (VA) has established patient education and multidisciplinary interventions to optimize engagement in T2DM selfmanagement and health behavior change. ${ }^{5}$

Traditional T2DM education programs aim to meet the American Diabetes Association (ADA) standards of medical care and include a T2DM educator and other allied health professionals. ADA Standard $1.2 \mathrm{em}$ phasizes "productive interactions between a prepared, proactive care team and an informed, activated patient." ${ }^{6}$ Thus, to attain ADA accreditation, educational programs require instructors to teach about T2DM while engaging patients to help them set and achieve recommended changes. The requirements emphasize setting specific goals, (ie, eating wisely, being physically active, monitoring blood sugars or taking medications). The care team also helps to identify barriers, and at a required follow-up class, patients evaluate how well they met goals and make modifications if needed. The impact of traditional patient education programs to improve glycemic levels is well established. ${ }^{7}$ Importantly, veterans with comorbid mental health conditions may not experience the same beneficial outcomes if or when they participate in traditional diabetes or selfmanagement programs. ${ }^{8,9}$ Veterans with T2DM may be particularly vulnerable to chronic stress and effects of comorbid mental health diagnoses. ${ }^{10}$ Furthermore, when individuals experience T2DM-related distress, associations with poor health outcomes, including elevated hemoglobin $\mathrm{A}_{1 \mathrm{c}}\left(\mathrm{Hb}_{\mathrm{lc}}\right)$, are observed independent of depression. ${ }^{11}$

Health psychology services integrate into medical settings and strive to reach veterans who may not engage in traditional mental health clinical offerings. ${ }^{12}$ These 
collaborative interventions focus less on diagnostic or screening procedures and more on a patient's understanding of illness and ability and willingness to carry out treatment regimens. Given the significant roles of distress and co-occurring conditions, health psychology services further aim to provide psychoeducation about stress management in order to explore and enhance motivation for making a wide range of health behavior changes.

The purpose of this study was to evaluate baseline and follow-up $\mathrm{HbA}_{1 \mathrm{c},}$, weight, and psychosocial measures, namely, healthrelated self-efficacy and T2DM-related distress among a small sample that engaged in integrated health psychology services. The focus of this evidence-based psychotherapy service was to improve T2DM selfcare and physical health. The participants were offered cognitive and behavioral strategies for setting and meeting personalized T2DM self-management goals. Importantly, motivational interviewing was used throughout to adapt to the participants' preferences and needs as well as to maintain engagement.

\section{METHODS}

Primary care providers referred veterans with T2DM to the Health Psychology service at VA Ann Arbor Healthcare System (VAAAHS). A T2DM diagnosis was verified through electronic health record review. Most common referrals included addressing coping with chronic illness and improving glycemic levels. Veterans were invited to participate in a program evaluation project to monitor healthrelated changes. All participants provided written informed consent and did not receive incentive or payment for participating. The VAAAHS Institutional Review Board reviewed and approved this study.

\section{Intervention}

Veterans met individually with a health psychologist or health psychology trainee to create personalized health and behavioral goals for improving T2DM selfmanagement, overall health, and psychological well-being. This intervention included motivational interviewing, SMART (specific, measurable, action-oriented, realistic, timely) goal setting, behavioral activation, acceptance of T2DM-related physical changes, prob-
TABLE Descriptive Statistics for the Demographic and Health Characteristics at Baseline $(\mathrm{N}=13)$

\begin{tabular}{|c|c|c|}
\hline Variables & Result & Range \\
\hline Age, mean (SD), y & $62.8(7.5)$ & $49-74$ \\
\hline \multicolumn{3}{|l|}{ Race/ethnicity, No. (\%) } \\
\hline White & $9(68.2)$ & \\
\hline African American & $3(23.1)$ & \\
\hline Hispanic/Other & $1(7.7)$ & \\
\hline Male, No. (\%) & $12(92.3)$ & \\
\hline High school diploma, No. (\%) & $9(69.2)$ & \\
\hline Time since diabetes diagnosis, mean (SD), y & $12.4(8.0)$ & $3-21$ \\
\hline Weight, mean (SD), lb & $259.9(67.6)$ & $168.0-449.7$ \\
\hline Body mass index, mean (SD) & $35.7(7.5)$ & $24.2-52.1$ \\
\hline Hemoglobin $A_{1 c}$, mean (SD), \% & $8.3(1.6)$ & $6.2-11.1$ \\
\hline Prescribed insulin, No. (\%) & $9(76.9)$ & \\
\hline Low-density lipoprotein cholesterol, mean (SD), mg/dL & $79.5(35.6)$ & $25-141$ \\
\hline Systolic blood pressure, mean (SD), mm Hg & $140.5(20.9)$ & $115-183$ \\
\hline Diastolic blood pressure, mean (SD), $\mathrm{mm} \mathrm{Hg}$ & $72.4(14.2)$ & $58-109$ \\
\hline Comorbid mental health conditions, mean (SD), No. & $1.6(1.0)$ & $0-3$ \\
\hline
\end{tabular}

lem-solving therapy, challenging maladaptive disease-related cognitions, and incorporating values to help find motivation for change. Interventionists took a flexible approach and met in-person in primary or specialty care clinics, over the phone, and through telehealth 1 to 4 times a month, meeting participant preferences, with sessions typically ranging from 45 to 60 minutes. The goal of the study was to disseminate and implement evidence-based behavioral change strategies into a multidisciplinary team format without excluding veterans who would benefit from receiving active treatment. Due to this translational approach, a control group was not included for comparison.

\section{Data Collection}

Participants completed study measures at the beginning and end of the T2DM-focused intervention sessions. Demographic variables collected included age, sex, race/ethnicity, 
FIGURE Baseline and Follow-up Changes in Health-Related Measurements and Psychosocial Variables

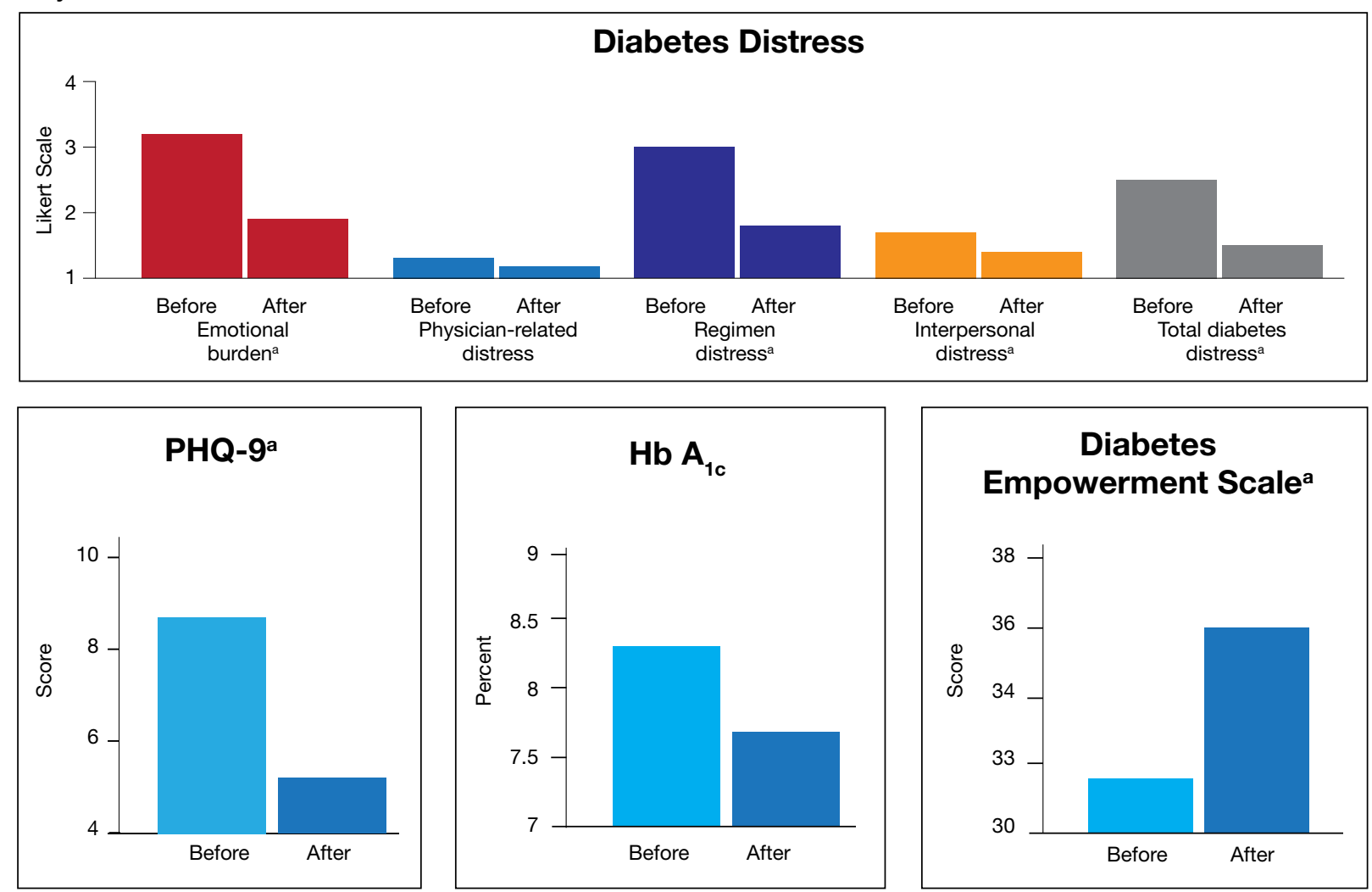

Abbreviations: $\mathrm{Hb} \mathrm{A} \mathrm{A}_{1 \mathrm{c}}$, Hemoglobin $\mathrm{A}_{1 \mathrm{c}} ; \mathrm{PHQ}-9$, Patient Health Questionnaire 9-item. a $P<.05$

highest educational attainment, and whether a veteran was prescribed insulin, service connected for T2DM, concurrent enrollment in other educational programs, and time since T2DM diagnosis. Measures were selected based on their relevance to T2DM psychosocial care and diabetes health outcomes. ${ }^{13}$

Body mass index, low-density lipoprotein cholesterol, blood pressure (BP), $\mathrm{HbA}_{1 \mathrm{c}}$ within 3 months of the pre- and postmeasures were collected by reviewing medical records. T2DM complications were collected by self-report, and comorbid physical and mental health conditions were collected by review of the most recent primary care note. The Diabetes Empowerment Scale-Short Form (DES-SF) is a well-validated measure that was used to measure T2DM-related psychosocial self-efficacy. ${ }^{14}$ Scores ranged from 8 to 40 with higher scores indicating higher diabetes T2DM empowerment. The $\mathrm{Pa}$ tient Health Questionnaire 9-item (PHQ-9) was used to assess the frequency of somatic (fatigue, appetite, psychomotor) and cognitive symptoms (anhedonia, low mood) of depression over the past 2 weeks. ${ }^{15}$ The Generalized Anxiety Disorder 7-item (GAD7) was used to assess the frequency of common anxiety symptoms, including feelings of worry, difficulty controlling worry, and trouble relaxing. ${ }^{16}$ Veterans were also asked to rate their general health on a 5-point Likert scale. Self-rated health is a well-established indicator of disability and risk of future T2DM complications in older adults. ${ }^{17,18}$ The Diabetes Distress Scale (DDS) was used to measure emotional burden, physicianrelated distress, regimen-related distress, and T2DM-related interpersonal distress. ${ }^{19}$ Scores $>2.0$ suggest clinical significant diabetes distress. ${ }^{20}$ Medication questionnaires were adapted from Wilson and colleagues, 2013. ${ }^{21}$

\section{Statistical Analyses}

Descriptive statistics, including mean and standard deviation (SD) or frequency 
distributions, as appropriate, were used to characterize the sample. For pre- and postintervention within-group comparisons, a paired samples Student $t$ test analysis was used to evaluate baseline and follow-up measures for statistically significant differences between continuous variables; scores also were evaluated for clinically meaningful change.

\section{RESULTS}

This sample $(\mathrm{N}=13)$ of older adults was predominately male, white, with $\mathrm{HbA}_{1 \mathrm{c}}$ $>7.0$, and prescribed insulin (Table). On average, participants were at higher risk for future complications due to high BP, hyperlipidemia, and BMI > 30.0. Regarding participation, veterans were seen for an average of 7.8 sessions (range, 4-13) with $46 \%$ service connected for T2DM. Of note, 4 veterans received other T2DM-specific self-management support within the same year of their participation with health psychology, such as attending a T2DM education class or T2DM shared medical appointment. ${ }^{22}$ Reliability in the current sample for the DES-SF was high (Cronbach $\alpha=0.90$ ), PHQ-9 was good (Cronbach $\alpha=0.81$ ), and GAD-7 was very good (Cronbach $\alpha=0.86$ ).

Among the 13 older adults, the most common T2DM-related complications included peripheral neuropathy $(\mathrm{n}=7)$, heart pain or heart attack $(n=5)$, and retinopathy ( $\mathrm{n}$ $=4)$. Recent primary care notes showed a mean (SD) 7 (2.2) comorbid chronic medical conditions with a high prevalence of cardiometabolic illnesses including hypertension, hyperlipidemia, obstructive sleep apnea, and a diagnosis of chronic pain. Eleven veterans were diagnosed with a mental health condition, including bipolar disorder, depression, anxiety, trauma-related disorder, and sleep disorders. Veterans reported high T2DM emotional distress (mean [SD] 3.1 [1.2]), moderate regimen-related distress (mean [SD] 2.9 [1.1]), and moderate total T2DM distress (mean [SD] 2.4 [0.7]). Physician distress (mean [SD] 1.3 [0.55]) and interpersonal T2DM distress (mean [SD] 1.6 [0.9]) subscales indicated little to no distress. The sample reported mild symptoms of depression (PHQ-9 mean [SD] 8.8 [4.6]); mild symptoms of anxiety
(GAD-7 mean, 7.1; SD, 4.4), and Diabetes Empowerment (mean, 31.2; SD, 6.0). Participants described missing an average of 2.4 days within the past 30 days of their T2DM oral medications.

Twelve veterans (92.7\%) completed the Follow-up questionnaires. The Figure illustrates statistically significant changes in patient-reported outcomes between baseline and follow-up. Clinically meaningful reductions were shown in total T2DM distress $\left(t_{11}=5.03, P<.01\right)$, T2DM emotional burden $\left(t_{11}=4.83, P=.01\right)$, and T2DM regimen-related distress $\left(t_{11}=5.14\right.$, $P<.01)$. There was a significant increase in T2DM self-efficacy $\left(t_{11}=0.32, P=.008\right)$ as well. A statistically significant reduction was seen in depressive symptoms $\left(t_{11}=\right.$ $2.22, P=.048$ ). While $\mathrm{HbA}_{1 \mathrm{c}}$ fell by .56 percentage points (standard error of the mean [SEM] , 31; $P=.10$ ), this change was not statistically significant. Follow-up analyses also showed a clinically, though not statistically, significant reduction in weight loss by $6.9 \mathrm{lb}$. (SEM, 3.8; $P=.20$ ), and reductions of generalized anxiety by 1.2 points (SEM, $1.4 ; P=.42$ ). Pre- and postanalyses did not show differences among self-rated health, physician-related burden, interpersonalrelated burden, and indicators of medication taking behavior.

\section{DISCUSSION}

This observational study evaluated change among patient-reported T2DM-specific and general distress measures and health outcomes among a small sample of veterans at VAAAHS medical center that engaged in an episode of individual care with health psychology. Statistically significant decreases were observed in T2DM-related distress. Noteworthy, these decreases were observed for the emotional burden and regimen subscales, and each of these was clinically meaningful, falling below a score of 2.0 on the T2DM-specific scale. This is important given that T2DM distress may interfere with the ability to understand and find motivation for engaging in health behavior change. Incorporating stress management interventions into interdisciplinary health programs has been demonstrated to improve not only levels of distress, but also other health outcomes, such as health related quality of life 
and cardiac events in heart disease. ${ }^{23}$ Thus, behavioral health interventions that incorporate cognitive-behavioral strategies to enhance distress-specific coping may prove important to include among individuals with T2DM

Reductions in T2DM-related distress also converged with increases observed in the T2DM empowerment scale. These significant improvements in perceived ability suggest increased self-efficacy and willingness to follow a daily T2DM regimen. This finding aligns with the social support literature that demonstrates how instrumental and other aspects of autonomous social support mediate improvements in healthrelated outcomes and reduced T2DM distress. ${ }^{24,25}$ Health psychology interventions strive to both provide social support as well as enhance participants' perceptions and use of existing support as a cognitivebehavioral strategy. Adding in assessments of social support could shed light on such mediating factors.

The ADA standards of care encourage heath care providers to engage patients in conversations in order to better understand the barriers of T2DM self-care. ${ }^{13}$ How to best support patients within a primary care multidisciplinary team remains unclear. ${ }^{26} \mathrm{~T} 2 \mathrm{DM}$ distress and negative reactions to T2DM, including symptoms of anxiety and depression, are common and may require specific referral to a mental health provider if repeated attempts at T2DM education do not improve self-management and illness biomarkers. ${ }^{27}$ Thus, integrating these providers and services within the medical setting aims to reach more veterans and potentially meet these standards of care. With our health psychology integrated services, clinically significant decreases in anxiety and statistically significant decreases in depressive symptoms were observed that approached "mild to no" symptoms. Although this was not measured formally, the veterans were not engaging in mental health specialty care historically or during the year of the health psychology intervention. This suggests that health psychology services helped bridge the gap and address these psychosocial needs within the small sample.

For clinical measures, modest decreases were observed for $\mathrm{HbA}_{1 \mathrm{c}}$ and weight. The authors recognize that these changes may not be optimal in terms of health status. A review of the specific patient-centered goals may illuminate this finding. For example, 1 participant had a goal to consume fewer sugary beverages and achieved this behavior change. Yet this change alone may not equate to actual weight loss or a lower $\mathrm{HbA}_{1 \mathrm{c}}$. Furthermore, in the context of T2DM-related distress, maintaining current weight and/or blood sugar levels may be a more realistic goal. An evaluation of the specific patient-oriented action goals and observed progress may be important outcomes to include in larger studies. Moreover, while not significant, the average $\mathrm{HbA}_{1 \mathrm{c}}$ decrease of about $1 \%$ is comparable with traditional T2DM education and should be considered in light of the sample's significant mental health comorbidities. While landmark intensive glucose control trials illustrate significant benefits in reductions of hyperglycemia and nonfatal cardiovascular disease, these reductions are associated with an approximate 2-fold risk of hypoglycemia. ${ }^{28-30}$ Thus, the focus on improved glycemic control has been criticized as lacking meaning to patients in contrast to preventing T2DM complications and persevering quality of life. ${ }^{31}$

\section{Limitations and Future Directions}

Noted limitations include small sample size, the range of time, and a broad number of sessions given that the intervention was tailored to each veteran. Conclusions drawn from a small sample may be influenced by individual outliers. Given cooccurring conditions and moderate levels of distress, all participants may benefit from additional support resources.

In addition to these considerations, having a comparison group could further strengthen the study as part of an observational database. A between-group comparison could help clinicians better understand what the interventions offer as well as some individual factors that relate to participation and success with behavior change. In the future, studies with a priori hypotheses could also consider the trajectories of weight and blood sugar levels for extended periods; for example, 6 months before the intervention and 6 months following. ${ }^{32}$ 
Given the complexity of comorbid mental health and chronic medical conditions in this sample, it also may be important to measure the relationships between chronic physical symptoms as an additional barrier for veterans to make health behavior changes.

\section{CONCLUSIONS}

The authors believe that the health psychology interventions offered important support and motivation for engagement in health behavior change that led to reduced distress in this patient group. It remains a challenge to engage veterans with psychiatric conditions in mental health care, and simultaneously for health care systems that strive to reduce costs and complications associated with chronic illness management. ${ }^{33}$ Aligned with these broader health care goals, the ADA aims to reduce complications and cost and improve outcomes for T2DM with guidelines requiring mental and behavioral health interventions. The authors believe that health psychology interventions are a personalized and feasible bridge to address engagement, illness-related distress while improving patient-satisfaction and T2DM self-management.

\section{Acknowledgments}

The authors thank the veterans who participated in the observational study. We thank the VA Ann Arbor Healthcare System Institutional Review Board. For instrumental support for health psychology integrated services, we acknowledge Adam Tremblay, MD, Primary Care Chief, and R.J. Schildhouse, MD, Acting Associate Chief of Staff, Ambulatory Care. The work was supported by the Ambulatory Care Service at the VA Ann Arbor Healthcare System and the VA Office of Academic Affiliations.

\section{Author affiliations}

Naomi Kane is a Clinical Psychology Postdoctoral Fellow in behavioral medicine and postdeployment health at the New Jersey VA War Related IIIness and Injury Study Center in East Orange. Naomi Kane was previously a Psychology Intern; Lindsey Bloor is a Clinical Health Psychologist and the Health Behavior Coordinator; Jamie Michaels is a Registered Dietician and Certified Diabetes Educator; all at the VA Ann Arbor Healthcare System in Michigan. Lindsey Bloor is a Clinical Assistant Professor in Psychiatry at the University of Michigan Medical School in Ann Arbor.

\section{Author disclosures}

The authors report no actual or potential conflicts of interest with regard to this article.

\section{Disclaimer}

The opinions expressed herein are those of the authors and do not necessarily reflect those of Federal Practitioner, Frontline Medical Communications Inc., the US Government, or any of its agencies.
References

1. Liu $Y$, Sayam S, Shao $X$, et al. Prevalence of and trends in diabetes among veterans, United States, 2005-2014. Prev Chronic Dis. 2017;14(12):E135, 1-5. doi:10.5888/pcd14.170230

2. Yu W, Ravelo A, Wagner TH, et al. Prevalence and costs of chronic conditions in the VA health care system. Med Care Res Rev. 2003;60(3)(suppl):146S-167S. doi:10.1177/1077558703257000

3. American Psychological Association. Psychology and Health in Action. Updated 2016. Accessed February 10, 2021. https://www.apa.org/health/fall-2016-updates.pdf

4. The US Burden of Disease Collaborators. The state of US health, 1990-2016. JAMA. 2018;319(14):1444-1472. doi:10.1001/jama.2018.0158

5. Piette JD, Kerr E, Richardson C, Heisler M. Veterans Affairs research on health information technologies for diabetes self-management support. J Diabetes Sci Technol. 2008;2(1):15-23. doi:10.1177/193229680800200104

6. American Diabetes Association. 1. Improving care and promoting health in populations: Standards of Medical Care in Diabetes-2019. Diabetes Care. 2019;42(suppl 1):S7-S12. doi:10.2337/dc19-S001

7. Norris SL, Lau J, Smith SJ, Schmid CH, Engelgau MM. Self-management education for adults with type 2 diabetes. A meta-analysis of the effect on glycemic control. Diabetes Care. 2002;25(7):1159-1171. doi:10.2337/diacare.25.7.1159

8. Janney CA, Owen R, Bowersox NW, Ratz D, Kilbourne EA. Bipolar disorder influences weight loss in the nationally implemented MOVE! program for veterans. Bipolar Disord. 2015;17:87.

9. Piette JD, Kerr EA. The impact of comorbid chronic conditions on diabetes care. Diabetes Care. 2006;29(3):725-731. doi:10.2337/diacare.29.03.06.dc05-2078

10. Trief PM, Ouimette P, Wade M, Shanahan P, Weinstock RS. Post-traumatic stress disorder and diabetes: Co-morbidity and outcomes in a male veterans sample. J Behav Med. 2006;29(5):411-418. doi:10.1007/s10865-006-9067-2

11. Fisher L, Mullan JT, Arean P, Glasgow RE, Hessler D, Masharani U. Diabetes distress but not clinical depression or depressive symptoms is associated with glycemic control in both cross-sectional and longitudinal analyses. Diabetes Care. 2010;33(1):23-28. doi:10.2337/dc09-1238

12. Bohnert KM, Pfeiffer PN, Szymanski BR, McCarthy JF. Continuation of care following an initial primary care visit with a mental health diagnosis: differences by receipt of VHA Primary Care-Mental Health Integration services. Gen Hosp Psychiatry. 2013;35(1):66-70. doi:10.1016/j.genhosppsych.2012.09.002

13. Young-Hyman D, De Groot M, Hill-Briggs F, Gonzalez JS, Hood K, Peyrot M. Psychosocial care for people with diabetes: a position statement of the American Diabetes Association. Diabetes Care. 2016;39(12):2126-2140. doi:10.2337/dc16-2053

14. Anderson R, Fitzgerald J, Gruppen L, Funnell M, Oh $M$. The diabetes empowerment scale-short form (DES-SF). Diabetes Care. 2003;26(5):1641-1642. doi:10.2337/diacare.26.5.1641-a

15. Kroenke K, Spitzer RL, Williams JBW. The PHQ-9: validity of a brief depression severity measure. J Gen Intern Med. 2001;16(9):606-613. doi:10.1046/j.1525-1497.2001.016009606.x

16. Spitzer RL, Kroenke K, Williams JBW, Löwe B. A brief measure for assessing generalized anxiety disorder: the GAD-7. Arch Intern Med. 2006;166(10):1092-1097. doi:10.1001/archinte.166.10.1092

17. Pinquart M. Correlates of subjective health in older adults: a meta-analysis. Psychol Aging. 2001;16(3):414. doi:10.1037/0882-7974.16.3.414

18. Hayes AJ, Clarke PM, Glasziou PG, Simes RJ, Drury PL, Keech AC. Can self-rated health scores be used for risk prediction in patients with type 2 diabetes? Diabetes Care. 2008;31(4):795-797. doi:10.2337/dc07-1391

19. Polonsky WH, Fisher L, Earles J, et al. Assessing psy- 
chosocial distress in diabetes: development of the diabetes distress scale. Diabetes Care. 2005;28(3):626-631. doi:10.2337/diacare.28.3.626

20. Fisher L, Hessler DDM, Polonsky WH, Mullan J. When is diabetes distress meaningful?: Establishing cut points for the Diabetes Distress Scale. Diabetes Care. 2012;35(2):259-264. doi:10.2337/dc11-1572

21. Wilson IB, Fowler FJ Jr, Cosenza CA, et al. Cognitive and field testing of a new set of medication adherence selfreport items for HIV care. AIDS Behav. 2013;18(12):23492358. doi:10.1007/s10461-013-0610-1

22. Heisler M, Burgess J, Cass J, et al. The Shared Health Appointments and Reciprocal Enhanced Support (SHARES) study: study protocol for a randomized trial. Trials. 2017;18(1):239. doi:10.1186/s13063-017-1959-7

23. Blumenthal JA, Babyak MA, Carney RM, et al. Exercise, depression, and mortality after myocardial infarction in the ENRICHD Trial. Med Sci Sports Exerc. 2004;36(5):746-755. doi:10.1249/01.MSS.0000125997.63493.13

24. Lee AA, Piette JD, Heisler M, Rosland AM. Diabetes distress and glycemic control: the buffering effect of autonomy support from important family members and friends. Diabetes Care. 2018;41(6):1157-1163. doi:10.2337/dc17-2396

25. Baek RN, Tanenbaum ML, Gonzalez JS. Diabetes burden and diabetes distress: the buffering effect of social support. Ann Behav Med. 2014;48(2):1-11. doi:10.1007/s12160-013-9585-4

26. Jortberg BT, Miller BF, Gabbay RA, Sparling K, Dickinson WP. Patient-centered medical home: how it affects psychosocial outcomes for diabetes. Curr Diab Rep.
2012;12(6):721-728. doi:10.1007/s11892-012-0316-1

27. American Diabetes Association. Lifestyle management: standards of medical care in diabetes-2019. Diabetes Care. 2019;41(suppl 1):S38-S50. doi:10.2337/dc19-S005

28. UK Prospective Diabetes Study Group. Effect of intensive blood-glucose control with metformin on complications in overweight patients with type 2 diabetes. Lancet. 1998;352(9131):854-865.

29. The Diabetes Control and Complications Trial Research Group, Control TD, Trial C. The effect of intensive treatment of diabetes on the development and progression of long-term complications in insulin-dependent diabetes mellitus. The Diabetes Control and Complications Trial Research Group. N Engl J Med. 1993;329(14):977-986. doi:10.1056/NEJM199309303291401

30. Kelly TN, Bazzano LA, Fonseca VA, Thethi TK, Reynolds $\mathrm{K}, \mathrm{He}$ J. Systematic review: glucose control and cardiovascular disease in type 2 diabetes. Ann Intern Med. 2009;151(6):394-403. doi:10.1037/1072-5245.13.1.64

31. Yudkin JS, Lipska KJ, Montori VM. The idolatry of the surrogate. BMJ. 2012;344(7839):8-10. doi:10.1136/bmj.d7995

32. Lutes LD, Damschroder LJ, Masheb R, et al. Behavioral treatment for veterans with obesity: 24-month weight outcomes from the ASPIRE-VA Small Changes Randomized Trial. J Gen Intern Med. 2017;32(1):40-47. doi:10.1007/s11606-017-3987-0

33. Krejci LP, Carter K, Gaudet T. The vision and implementation of personalized, proactive, patient-driven health care for veterans. Med Care. 2014;52(12)(suppl 5):S5-S8. doi:10.1097/MLR.0000000000000226 